\title{
Bioinformatics of thymidine metabolism in Trypanosoma evansi: exploring nucleoside deoxyribosyltransferase (NDRT) as a drug target
}

\author{
Kandeel, M. ${ }^{1,2^{*}}$, Alhumam, M.N. ${ }^{3}$, Al-Taher, A. ${ }^{1}$ \\ ${ }^{1}$ Department of Biomedical Sciences, College of Veterinary Medicine, King Faisal University, Alahsa, 31982, Saudi Arabia \\ 2Department of Pharmacology, Faculty of Veterinary Medicine, Kafrelsheikh University, Kafrelsheikh, 35255, Egypt \\ ${ }^{3}$ College of Medicine, King Faisal University, Alahsa, 31982, Saudi Arabia \\ *Corresponding author: mkandeel@kfu.edu.sa
}

\section{ARTICLE HISTORY}

Received: 20 September 2020

Revised: 22 June 2021

Accepted: 22 June 2021

Published: 30 August 2021

\begin{abstract}
Trypanosoma evansi, the causative agent of surra or camel trypanosomiasis, is characterized by the widest geographic distribution and host range among the known trypanosomes. Its zoonotic importance and increasing evidence of drug resistance necessitate the discovery of new drug targets. The drug discovery process entails finding an exploitable difference between the host and the parasite. In this study, the thymidine metabolic pathways in camel and $T$. evansi were compared by analyzing their metabolic maps, protein sequences, domain and motif contents, phylogenetic relationships, and 3D structure models. The two organisms were revealed to recycle thymidine differently: performed by thymidine phosphorylase in camels (Camelus genus), this role in T. evansi was associated with nucleoside deoxyribosyltransferase (NDRT), a unique trypanosomal enzyme absent in camels. Thymidine in $T$. evansi seems to be governed by thymine through NDRT, whereas in camels, thymidine can be produced from thymidylate via 5 '-nucleotidase. As a result, NDRT may be a promising drug target against $T$. evansi.
\end{abstract}

Keywords: Bioinformatics; camel; Trypanosoma; protozoa; drug discovery.

\section{INTRODUCTION}

Decoding camel (Camelus genus) genome sequences (Jirimutu et al., 2012) will accelerate drug discovery studies against camel pathogens. One specific line of inquiry is investigating pyrimidine metabolic pathways, and finding unique differences in structure, function, sequence, or phylogeny associated with these pathways could contribute to identifying new drug targets. KEGG maps have been used (Ogata et al., 1998; Kanehisa et al., 2007; Kanehisa et al., 2016) to compare the enzymes involved in thymidine metabolism pathways in camels and $T$. evansi.

In this work, comprehensive bioinformatic tools were used to investigate enzyme sequences in camels and $T$. evansi and compare their domains and motif content, searching for similar proteins and identifying their phylogenetic relationships. We plotted the pathway of thymidine metabolism in the two organisms, providing bioinformatics evidence of unique differences between camels and $T$. evansi that could be exploited for future drug discovery processes.

\section{MATERIALS AND METHODS}

\section{Construction of the metabolic map}

The Kyoto Encyclopedia of Genes and Genomes (KEGG) website was used to retrieve the thymidine pathway maps.
The map was accessed at the following link (https:// www.genome.jp/kegg-bin/show_pathway?map00240). The structure of nucleotides and the pathways were written by ChemDraw software (Figure 1).

\section{Retrieval of protein sequences}

The NCBI protein database was used to obtain the sequences of camel enzymes (http://www.ncbi.nlm.nih.gov/protein), and the $T$. evansi protein sequences were retrieved from the Kinetoplastom genome project (http://tritrypdb.org/tritrypdb). The sequence files in FASTA format were stored and processed by multiple software programs, including the CLC main workbench, Geneious, and Genedoc. The accession numbers of the retrieved sequences are provided in Figures 2-5.

Basic local alignment search tool (BLAST)

A BLAST search was performed using the NCBI search engine (Madden, 2013). The BLAST tool was used to find orthologues with high similarity to the retrieved sequences by searching a database of non-redundant ( $\mathrm{nr}$ ) protein sequences using the default settings. The retrieved camel and $T$. evansi sequences were input into the protein BLAST server at (https://blast.ncbi.nIm.nih.gov/Blast.cgi?PROGRAM =blastp\&PAGE_TYPE=BlastSearch\&LINK_LOC=blasthome) using the FASTA sequences and searching the $\mathrm{nr}$ protein 
database and blastp algorithm.

\section{Alignment of multiple and pairwise protein sequences}

Clustal Omega was used to align the protein sequences (Sievers \& Higgins, 2014). The obtained alignment file was processed by the CLC main workbench. The aligned sequences were subjected to pairwise comparisons to determine the identity\% and the number of amino acid differences.

\section{Construction of phylogenetic tree}

The phylogenic tree was built from the obtained alignment files and visualized by the Dendroscope phylogenetic tree viewer (Huson et al., 2007) or CLC main workbench (Qiagen software, Hilden, Germany). The tree was constructed by the neighbor-joining method. Bootstrap analysis was performed and set to 100 replicates.

\section{Putative domain and motif search}

The NCBI domain prediction program available at (http:// www.ncbi.nlm.nih.gov/Structure/cdd/cdd.shtml) (MarchlerBauer et al., 2005) was used to map the domains and motifs of the retrieved proteins. Domains and motifs were also explored using a KEGG motif finder tool.

\section{Proteomic and genomic tools}

The tools available at ExPASy Proteomics (http://us.expasy. org/tools/) (Gasteiger et al., 2003) and (http://www.ebi.ac.uk/
Tools/) (Labarga et al., 2007) were used to analyze the protein sequences. The tools utilized comprised Clustal Omega for protein alignment, the Swiss Model for protein structure model construction, the Prosite for protein family and domain checking, and BLAST protein for the protein search. All analyses were run using the default parameters.

\section{Building structure model}

Based on the $T$. evansi sequence, a structure model was built using the Swiss Model server. The sequence of $T$. evansi NDRT was entered in FASTA format in SWISS-MODEL project mode (https://swissmodel.expasy.org/interactive).

\section{RESULTS}

The pyrimidine metabolic maps at KEGG were used to trace the thymidine pathways (Kanehisa \& Goto, 2000). In the de novo pathway, thymidine kinase converts thymidine to thymidine monophosphate (TMP). In catabolism, TMP is converted to thymidine by the action of 5 '-nucleotidase (Figure $1 \mathrm{~A}$ ). Further, thymidine is converted to thymine by thymidine/pyrimidine phosphorylase, whereas deoxyribosyltransferase catalyzes the interconversion of thymidine and thymine.

In the camel thymidine pathway, camels were expected to be devoid of NDRT (Figure 1B). Therefore, there is no interconversion between thymine and thymidine in camel pyrimidine metabolism. Thus, the sole source of thymidine

\section{A}
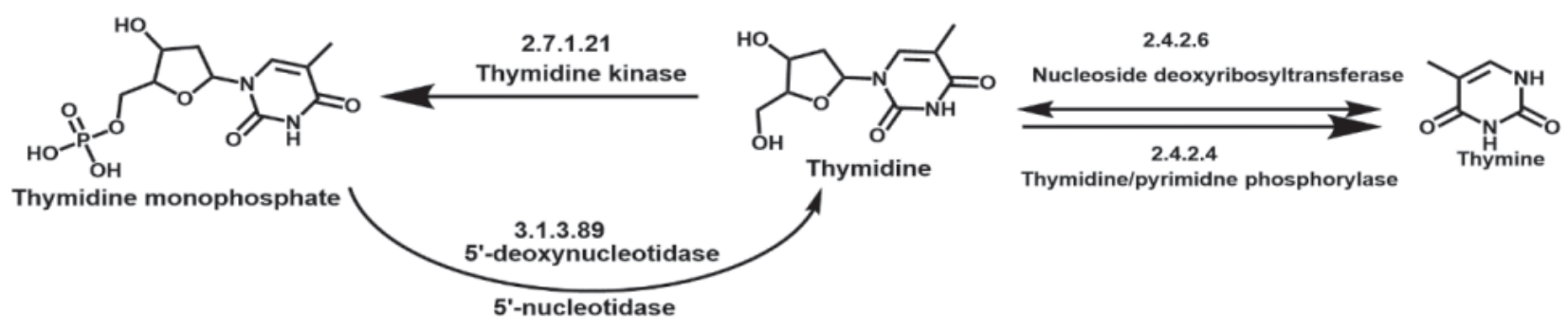

B
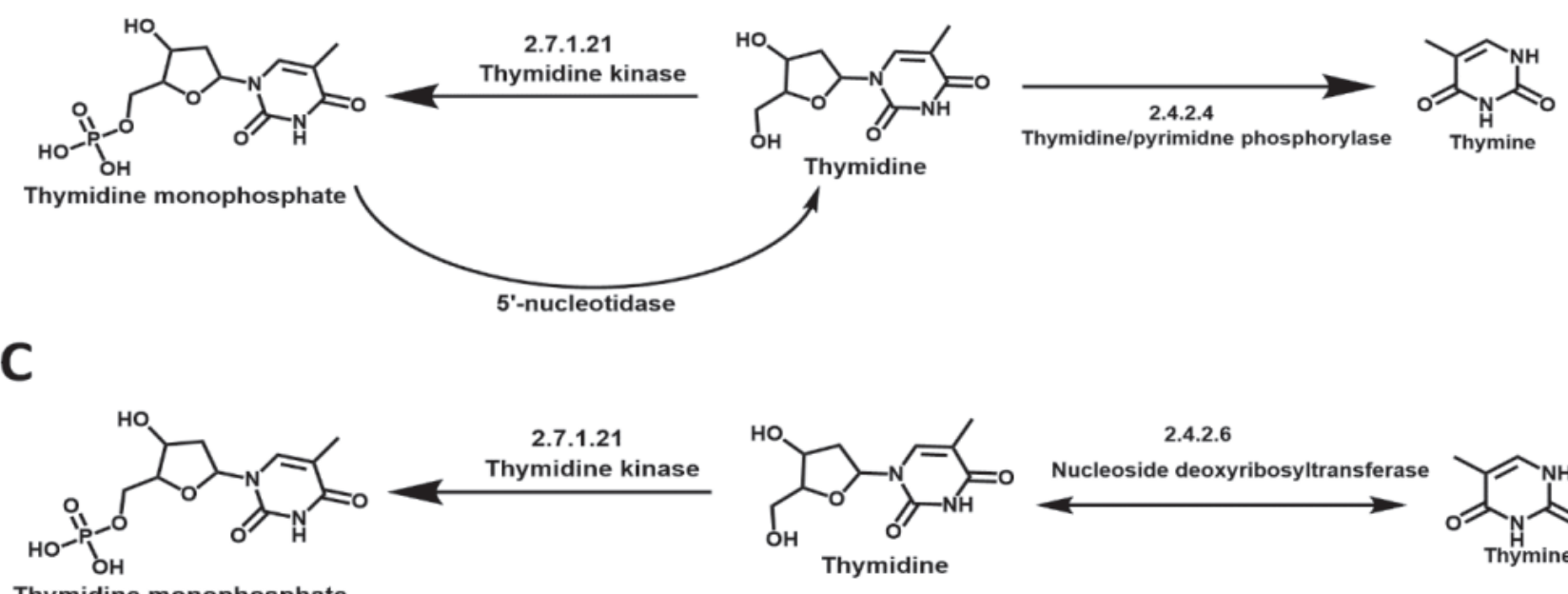

Thymidine monophosphate

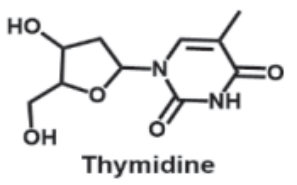

2.4.2.6

Nucleoside deoxyribosyltransferase
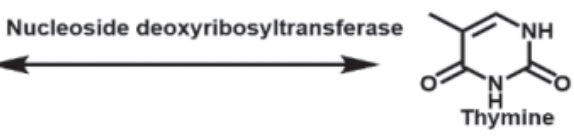

Figure 1. Thymidine metabolic pathways. A) The proposed general metabolic pathways of thymidine. B) The proposed metabolic pathways of thymidine in camels. C) The proposed metabolic pathways of thymidine in Trypanosoma evansi. 
in camels is from TMP by the action of 5'-nucleotidase or potentially by the transport of ready-formed thymidine from outside the cells.

In contrast, in the $T$. evansi metabolic map, the enzymes 5 '-nucleotidase and thymidine/pyrimidine phosphorylase were not confirmed, suggesting notable differences in thymidine metabolism. TMP cannot be converted to thymidine in $T$. evansi due to the absence of $5^{\prime}$-nucleotidase. The sole metabolic source of thymidine could be from thymine by the action of nucleoside deoxyribosyltransferase, which is absent in camels (Figure 1C). The list of enzymes of the thymidine pathway and the predicted content in camels and $T$. evansi are provided in Tables 1-3.

\section{Camel thymidine/pyrimidine phosphorylase (TP)}

Thymidine phosphorylase (EC 2.4.2.4, TP, pyrimidine phosphorylase, thymidine orthophosphate deoxyribosyltransferase, pyrimidine deoxynucleoside phosphorylase, or thymidine:phosphate deoxy-D-ribosyltransferase) is a glycosyltransferase that stimulates reversible dephosphorylation of thymidine phosphate supplying thymine and 2-deoxy-D-ribose 1-phosphate. In addition, it catalyzes deoxyribosyltransferase reactions catalyzed by NDRT in various tissues. The enzyme can also convert thymidine to thymine, which is not used by trypanosomes (Al Chalabi \& Gutteridge, 1977; Elamin et al., 2016). The properties of the phosphorylases differ significantly from prokaryotes to those of mammalian animals. For example, in Giardia lamblia, mainly dependent on salvage synthesis for its pyrimidine requirements, uridine, deoxyuridine, and thymidine are phosphorylated with one broad-spectrum nucleoside phosphorylase, which can also utilize uracil and thymine as substrates (Lee et al., 1988). Complicated infection of cancer tissues or cells with mycoplasmas reduced the efficiency of anticancer and antiviral nucleoside analogbased therapies due to the presence of mycoplasma TP. In Mycoplasma pneumoniae cultures, impaired activity of TP provoked elevated uptake and inclusion of deoxyuridine and uracil, but thymidine uptake was not affected. Thus, the enzymes of the mycoplasma nucleotide synthesis pathway are prospective antibiotic targets (Wang et al., 2014). In Trypanosoma cruzei, phosphorylase activity was detected but was more specific to uridine phosphorylase without specificity to thymidine or purine phosphorylase (Silva et al., 2011, 2012).

The obtained sequence of camel TP was very short and of a lower-quality protein than that of human TP. The recorded sequence of camel TP was 202 amino acids, only $18.44 \%$ similar to the 482 amino acid human TP. Similarly, of the TP sequences in the three camel species (dromedary, Bactrian, and wild camels), Camelus ferus had the shortest length, 162 amino acids (Figure 2). Comparing TPs from different

Table 1. All enzymes known to be involved in metabolic pathways of thymidine

\begin{tabular}{ll}
\hline ID (E.C. no.) & Enzyme \\
\hline 2.7.1.21 & Thymidine kinase \\
3.1 .3 .89 & 5'-deoxynucleotidase; 2 '-deoxyribonucleoside \\
& 5'-monophosphate phosphohydrolase \\
3.1 .3 .5 & 5'-nucleotidase; uridine 5'-nucleotidase \\
2.4 .2 .6 & nucleoside deoxyribosyltransferase \\
2.4 .2 .4 & thymidine phosphorylase; pyrimidine phosphorylase
\end{tabular}

Table 2. The expected enzymes involved in metabolic pathways of thymidine in camels

\begin{tabular}{ll}
\hline ID (E.C. no.) & Enzyme \\
\hline 2.7 .1 .21 & Thymidine kinase \\
3.1 .3 .5 & 5'-nucleotidase; uridine 5'-nucleotidase \\
2.4 .2 .4 & thymidine phosphorylase; pyrimidine phosphorylase
\end{tabular}

Table 3. The expected enzymes involved in metabolic pathways of thymidine in Trypanosoma evansi

\begin{tabular}{ll}
\hline ID (E.C. no.) & Enzyme \\
\hline 2.7 .1 .21 & Thymidine kinase \\
2.4 .2 .6 & nucleoside deoxyribosyltransferase \\
\hline
\end{tabular}

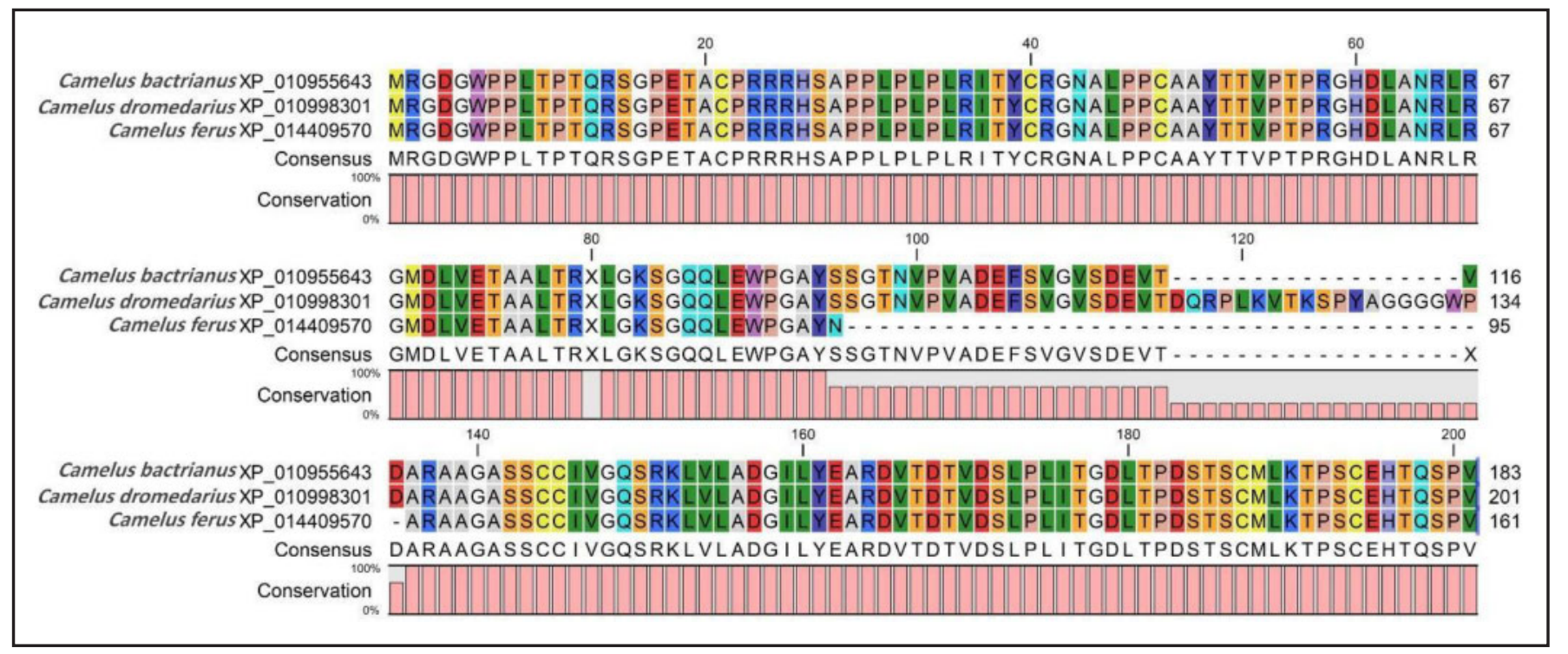

Figure 2. Multiple sequence alignment of dromedary and Bactrian camels thymidine/pyrimidine phosphorylase. 
prokaryotic and eukaryotic sources (Figure 3) showed a general low similarity rate from $9.5-41.8 \%$ among the tested species. Due to this difference, the camel TPs formed a monophyletic group that shared a common origin with the prokaryotic TPs and not the vertebrate TPs (Figure 4). The motif search retrieved zero hits using the motif finder and the pfam and prosite prediction tools, while a phosphorylase domain was predicted by the NCBI conserved domain search tool.

\section{Nucleoside deoxyribosyltransferase (NDRT) in T. evansi}

This enzyme was found to be unique for $T$. evansi and not present in camels, suggesting its use as a safe drug target. After searching the gene databases, NDRT could be predicted in some protozoa and bacteria, e.g., lactobacillus, Leishmania spp., and trypanosomes. A previous study investigated the crystal structure of $T$. brucei NDRT and found that its structure is highly similar to NDRT from Lactobacillus helveticus (Bosch et al., 2006). In the previous study, several crystal-bound

\begin{tabular}{|c|c|c|c|c|c|c|c|c|c|c|c|c|c|c|}
\hline & & 1 & 2 & 3 & 4 & 5 & 6 & 7 & 8 & 9 & 10 & 11 & 12 & 13 \\
\hline Escherichia coli VAX51408 & 1 & & 22 & 283 & 261 & 263 & 330 & 326 & 417 & 318 & 327 & 415 & 415 & 419 \\
\hline Salmonella enterica RXM16575 & 2 & 95.00 & & 281 & 263 & 266 & 332 & 327 & 418 & 320 & 329 & 415 & 415 & 419 \\
\hline Corynebacterium pseudotuberculosis ANH23382 & 3 & 39.40 & 39.83 & & 278 & 279 & 326 & 326 & 414 & 326 & 332 & 419 & 418 & 424 \\
\hline Staphylococcus aureus RIZ63567 & 4 & 41.48 & 41.03 & 40.47 & & 172 & 334 & 328 & 432 & 320 & 328 & 410 & 411 & 414 \\
\hline Clostridium tetani SJZ79245 & 5 & 41.03 & 40.36 & 40.26 & 60.73 & & 332 & 322 & 425 & 320 & 321 & 412 & 413 & 416 \\
\hline Homo sapiens ADP91533 & 6 & 34.26 & 33.86 & 34.80 & 32.39 & 33.20 & & 85 & 192 & 101 & 96 & 424 & 425 & 427 \\
\hline Oryctolagus cuniculus XP_002723933 & 7 & 34.41 & 34.21 & 34.67 & 32.92 & 34.69 & 82.44 & & 202 & 100 & 98 & 420 & 421 & 423 \\
\hline Equus cabal/lus XP_023487342 & 8 & 29.08 & 28.91 & 29.35 & 26.15 & 27.35 & 67.18 & 65.47 & & 226 & 226 & 524 & 525 & 527 \\
\hline Mus musculus NP_612175 & 9 & 34.84 & 34.43 & 34.27 & 33.33 & 33.88 & 79.18 & 79.25 & 61.37 & & 38 & 414 & 415 & 417 \\
\hline Rattus norvegicus NP_001012122 & 10 & 33.94 & 33.54 & 34.00 & 32.65 & 34.49 & 80.25 & 79.79 & 61.37 & 92.05 & & 420 & 421 & 423 \\
\hline Camelus bactrianus XP_010955643 & 11 & 10.17 & 10.17 & 9.50 & 10.48 & 10.24 & 14.17 & 14.46 & 12.08 & 14.64 & 14.29 & & 19 & 23 \\
\hline Camelus dromedarius XP_010998301 & 12 & 10.56 & 10.56 & 10.11 & 10.65 & 10.41 & 14.31 & 14.60 & 12.21 & 14.78 & 14.43 & 90.59 & & 41 \\
\hline Camelus ferus XP_014409570 & 13 & 8.71 & 8.71 & 7.83 & 9.01 & 8.77 & 13.03 & 13.32 & 11.13 & 13.49 & 13.14 & 87.50 & 79.70 & \\
\hline
\end{tabular}

Figure 3. The sequence comparison statistics of thymidine/pyrimidine phosphorylase. The upper right diagonal region explains the number of differences between two sequences, while the lower left diagonal region explains the \% of identity between two sequences.

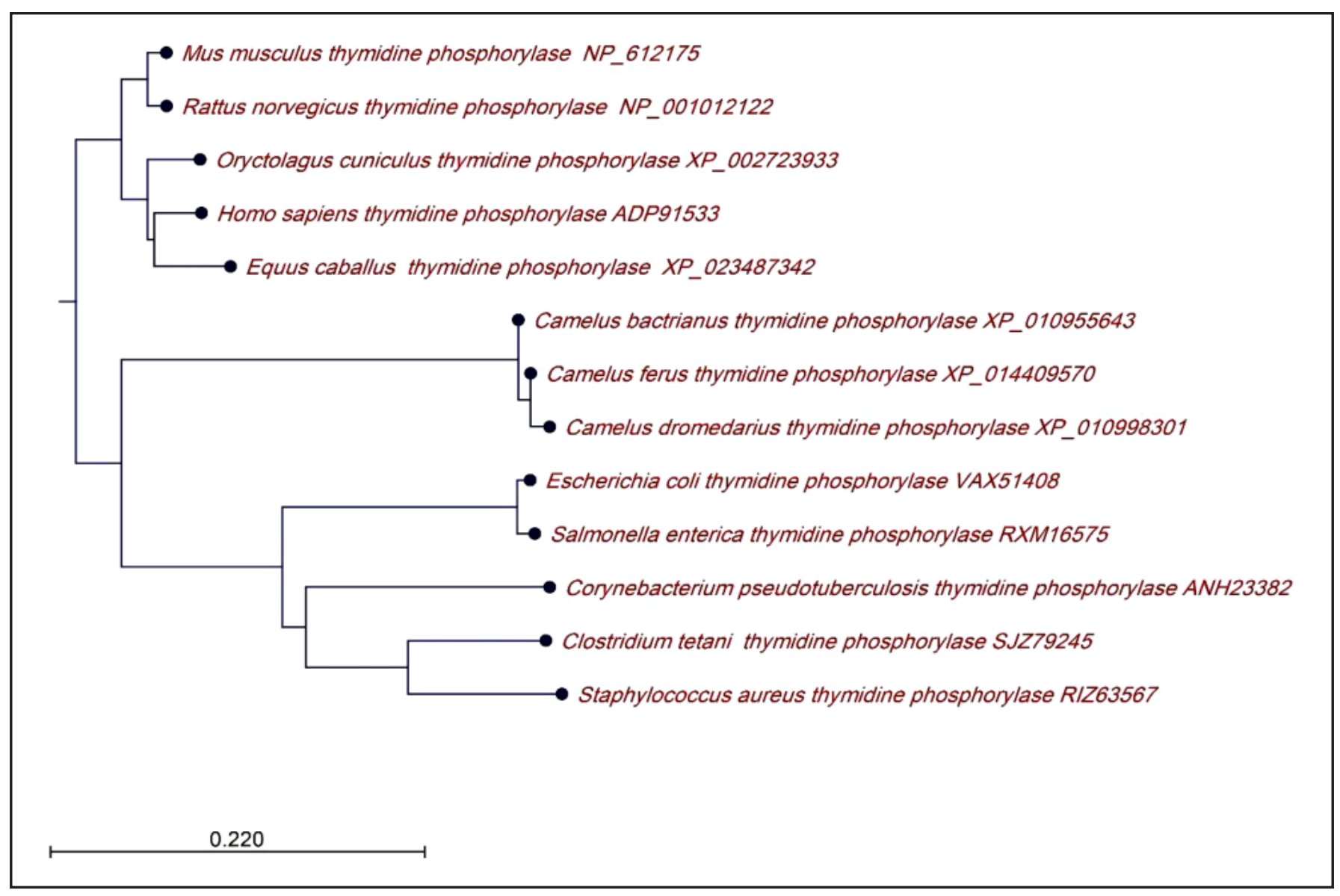

Figure 4. Phylogram of camel thymidine/pyrimidine phosphorylase in relation to a set of prokaryotic and eukaryotic organisms. 
compounds tested against the blood forms of $T$. brucei demonstrated weak inhibition of parasite growth, with IC50 values above $100 \mu \mathrm{M}$.

Sequence comparisons between T. evansi, Lactobacillus fecum, and Enterococcus fecum NDRTs revealed 17.68-21.21\% sequence similarity (Figure 5 ). Comparison of $T$. evansi and T. brucei NDRTs revealed $100 \%$ similarity. This finding may account for the observed similarity in the common features in the published structure, function, and inhibition.

The models for $T$. evansi NDRT were built using the Swiss Model server (Waterhouse et al., 2018). The model of $T$. evansi was predicted based on the deposited PDB ID 2a0k, which is the NDRT from $T$. brucei (Figure 6). The T. evansi NDRT modeled in dimer form with a monomer is 151 amino acids in length and composed of three beta sheets and five alpha helices. The dimer composition and interface are similar to the previously reported $T$. brucei structure (Bosch et al., 2006). The modeling statistics comprised $99 \%$ coverage, 0.6 sequence similarity, and $98 \%$ sequence identity.

\section{DISCUSSION}

The blood-protozoa $T$. evansi is the causative agent for surra or camel trypanosomiasis, a devastating disease in camels and other animals. T. evansi is characterized by the widest trypanosome geographic distribution and a large number of

\begin{tabular}{|c|r|r|r|r|} 
Lactobacillus fecum VEF34489 & & 1 & 2 & 3 \\
\cline { 2 - 5 } Enterococcus fecum VEF84603 & \multicolumn{1}{|c|}{2} & & 127 & 144 \\
\cline { 2 - 5 } Trypanosoma evansi & 3 & 17.12 & & 135 \\
\cline { 2 - 5 } & & &
\end{tabular}

20

Lactobacillus fecum VEF34489 MTKQKTVYFGAG - -WFTETQNKA YKDAMSALNANPTIDL 37 Enterococcus fecum VEF84603 MSQ - - I I LAGP - FFSEEQIDRVSRIIEKALEENKT VT - 33 Trypanosoma evansi MRK - - IYIIAGPAVFNPDMGASYYNKVRELLKKENVMPL 36

Consensus MXK - - I YXAGP - F F XEXQXXXYXXXXXALXXNXTXXL Conservation ${ }_{0 \%}^{100 \%}$ 40

Lactobacillus fecum VEF34489 ENSYVPLQNQYKDIRVDEHPEYLHDKEWAQATYNGDLVG 76

Enterococcus fecum VEF84603 - SFYSPRHHQESNYE - . - . - LFSAGWAQEVYEKDMEE 64

Trypanosoma evansi .... IPTDNEATG .................... ALIRQKNIQM 56

Consensus ${ }_{100 \%}$ - XXYXPXXNQXXXXX ........ L XXXXWAQXXYXKDXXX

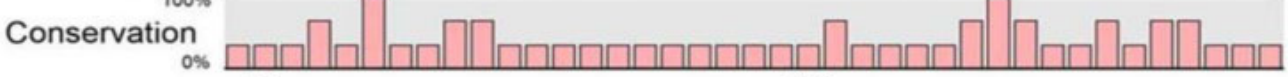

\%\% $\frac{\mathrm{ar}}{80} 100$

Lactobacillus fecum VEF34489 II KTSDIMLG - V - - YVPKEEDVG ÍLMELGYAMSQGKYVLL 112

Enterococcus fecum VEF84603 LTNAEFVVA - ILDFEHQTIDPGTAYELGVATMLKKPM I I 102

Trypanosoma evansi IKDCDAVIADLSPERGHEPDCGTAFEVGYAAALNKMVLT 95

Consensus ${ }_{100 \%}$ I KXXDXVXA - XXXFXXXEXDXGTAXELGYAXXLXKXVLX

Conservation

o\% $\frac{1}{120}$

120

140

Lactobacillus fecum VEF34489 VIPD - - - - ELYGESI - - - NLMSWGV - ADNVIKMSE - LA 141

Enterococcus fecum VEF84603 VQEE - . . . . TV - . - PT - . - NLMI TQS - LHTYLKSDQAVR 129

Trypanosoma evansi FTSDRRNMREKYGSEVDKDNLRVEGEGLPENLMLYDGVE 134

Consensus ${ }_{100 \%}$ VXXD - - . - EXYGXXX - - N L MXXGX - LXXXLKXXXXVX

Conservation

$0 \% \frac{\square \square}{160}$

160

Lactobacillus fecum VEF34489 TFDFNRPRYNFYDGAV - - Y 158

Enterococcus fecUm VEF84603 EYDFETLPVETYVGEY - - L 146

Trypanosoma evansi VFDSFESAFKYFLANFPSK 153

Consensus ${ }_{100 \%}$ XFDFXXXXXXXYXGXX- - X

Conservation

\%

Figure 5. Multiple sequence alignment of Trypanosoma evansi, Lactobacillus fecum and Enterococcus fecum nucleoside deoxyribosyltransferase. The upper panel represents sequence comparison statistics. The upper right diagonal region explains the number of differences between two sequences, while the lower-left diagonal region explains the \% of identity between two sequences. 


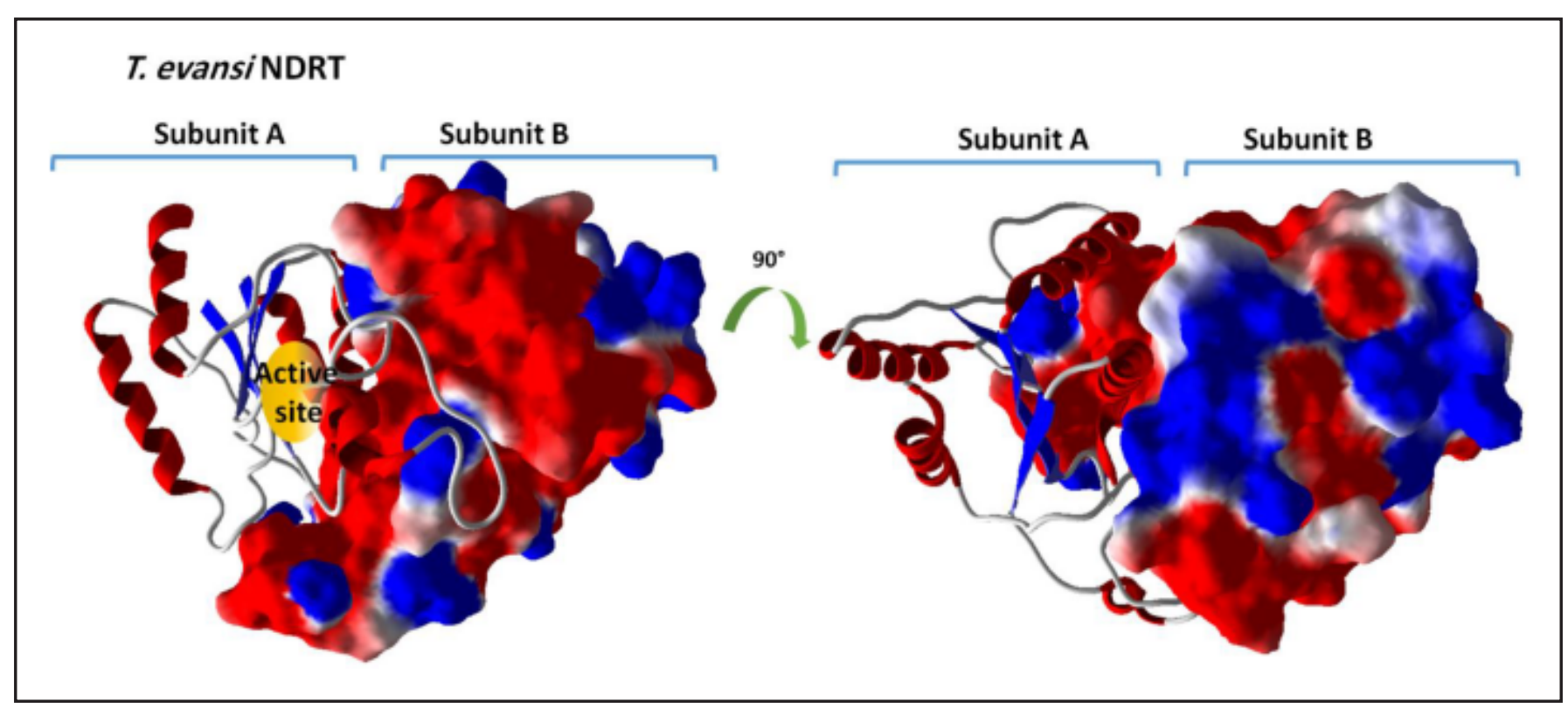

Figure 6. Molecular model of T. evansi NDRT. The model was built by using SwissModel.

hosts, including camels, large and small ruminant farm animals, bats, equines, pigs, carnivores, deer, gazelles, and elephants (Desquesnes et al., 2013). The recent human infections of humans with $T$. evansi has increased its public health importance (Joshi et al., 2005; Vanhollebeke et al., 2006).

The discovery of new drug targets against $T$. evansi is essential to combat this ubiquitous, zoonotic infection. The ideal target is present in the parasite and absent in the host. Recently, we have introduced several new antimicrobial targets, which were investigated using bioinformatics tools to elucidate the differences between the host and microbial proteins (Kandeel \& Kitade, 2011a, 2011b; Kato et al., 2012; Noguchi et al., 2013; Kandeel et al., 2020; Kandeel \& Al-Taher, 2020a, 2020b, 2021). In this context, pyrimidine enzymes are indispensable for life due to their role in nucleic acid synthesis.

NDRT was found to an important drug target in $T$. brucei (Bosch et al., 2006). Additionally, due to its efficient broadspectrum activity, NDRT has been used as a catalyst in the industrial synthesis of nucleotides (Fresco-Taboada et al., 2013; Crespo et al., 2017) and antiviral drugs (Hanrahan \& Hutchinson, 1992). In this study, NDRT was confirmed in $T$. evansi, and by bioinformatics tools, it was not detected in camels. This finding reveals NDRT as an attractive drug target against $T$. evansi. Future drug discovery studies targeting $T$. evansi NDRT are expected to yield safe and specific inhibitory compounds.

\section{CONCLUSION}

By analyzing the thymidine pathways in both camels and $T$. evansi, we could predict the differences in nucleoside recycling. The recycling of thymidine nucleosides in camels is governed by thymidine phosphorylase, absent in $T$. evansi. Similarly, the thymidine recycling in $T$. evansi is performed by NDRT, an enzyme unique to this protozoan, indicating its importance as a drug target in $T$. evansi.

\section{ACKNOWLEDGMENTS}

The authors acknowledge the financial support of this project by King Abdul-Aziz City for Science and Technology (KACST), Basic Research Programs, National Transformation Program, under Research and Development Grants Program for National Research Institutions and Centers (GPURC), Kingdom of Saudi Arabia (Grant No. 2-17-04-004-0001).

\section{Conflict of Interest}

The authors declare that they have no conflict of interests.

\section{REFERENCES}

Al Chalabi, K. \& Gutteridge, W.E. (1977). Catabolism of deoxythymidylate in some trypanosomatids. Parasitology 74: 299-312. https://doi.org/10.1017/s0031182000047922

Bosch, J., Robien, M.A., Mehlin, C., Boni, E., Riechers, A., Buckner, F.S., Van Voorhis, W.C., Myler, P.J., Worthey, E.A. \& DeTitta, G. (2006). Using fragment cocktail crystallography to assist inhibitor design of Trypanosoma brucei nucleoside 2-deoxyribosyltransferase. Journal of Medicinal Chemistry 49: 5939-5946. https://doi.org/10.1021/jm060429m

Crespo, N., Sánchez-Murcia, P.A., Gago, F., Cejudo-Sanches, J., Galmes, M.A., Fernández-Lucas, J. \& Mancheño, J.M. (2017). 22 -Deoxyribosyltransferase from Leishmania mexicana, an efficient biocatalyst for one-pot, one-step synthesis of nucleosides from poorly soluble purine bases. Applied Microbiology and Biotechnology 101: 7187-7200. https:// doi.org/10.1007/s00253-017-8450-y

Desquesnes, M., Holzmuller, P., Lai, D.H., Dargantes, A., Lun, Z.R. \& Jittaplapong, S. (2013). Trypanosoma evansi and surra: A review and perspectives on origin, history, distribution, taxonomy, morphology, hosts, and pathogenic effects. BioMed Research International 2013: 194176. https://doi.org/10.1155/2013/194176

Elamin, Y.Y., Rafee, S., Osman, N., O Byrne, K.J. \& Gately, K. (2016). Thymidine phosphorylase in cancer; enemy or friend? Cancer Microenvironment 9: 33-43. https://doi.org/ 10.1007/s12307-015-0173-y 
Fresco-Taboada, A., de la Mata, I., Arroyo, M. \& FernándezLucas, J. (2013). New insights on nucleoside 22 deoxyribosyltransferases: a versatile biocatalyst for onepot one-step synthesis of nucleoside analogs. Applied Microbiology and Biotechnology 97: 3773-3785. https:// doi.org/10.1007/s00253-013-4816-y

Gasteiger, E., Gattiker, A., Hoogland, C., Ivanyi, I., Appel, R.D. \& Bairoch, A. (2003). ExPASy: The proteomics server for indepth protein knowledge and analysis. Nucleic Acids Research 31: 3784-3788. https://doi.org/10.1093/nar/gkg563

Hanrahan, J.R. \& Hutchinson, D.W. (1992). The enzymatic synthesis of antiviral agents. Journal of Biotechnology 23: 193-210. https://doi.org/10.1016/0168-1656(92)90092-n

Huson, D.H., Richter, D.C., Rausch, C., Dezulian, T., Franz, M. \& Rupp, R. (2007). Dendroscope: An interactive viewer for large phylogenetic trees. BMC Bioinformatics 8: 460. https://doi.org/10.1186/1471-2105-8-460

Jirimutu, Wang, Z., Ding, G., Chen, G., Sun, Y., Sun, Z., Zhang, H., Wang, L., Hasi, S. \& Zhang, Y. (2012). Genome sequences of wild and domestic bactrian camels. Nature Communications 3: 1202. https://doi.org/10.1038/ncomms2192

Joshi, P.P., Shegokar, V.R., Powar, R.M., Herder, S., Katti, R., Salkar, H.R., Dani, V.S., Bhargava, A., Jannin, J. \& Truc, P. (2005). Human trypanosomiasis caused by Trypanosoma evansi in India: the first case report. The American Journal of Tropical Medicine and Hygiene 73: 491-495. https://doi.org/ 10.4269/ajtmh.2005.73.491

Kandeel, M. \& Al-Taher, A. (2020a). Deoxyuridine 5Monophosphate (dUMP) metabolising enzyme and the bifunctional dihydrofolate reductase-thymidylate synthase in camels and Trypanosoma evansi. Journal of Camel Practice and Research 27: 185-192. https://doi.org/10.5958/ 2277-8934.2020.00027.2

Kandeel, M. \& Al-Taher, A. (2020b). Metabolic drug targets of the cytosine metabolism pathways in the dromedary camel (Camelus dromedarius) and blood parasite Trypanosoma evansi. Tropical Animal Health and Production 52: 3337-3358. https://doi.org/10.1007/s11250-020-02366-8

Kandeel, M. \& Al-Taher, A. (2021). Molecular landscapes of deoxyuridine 5 '-triphosphatase (dUTPase) as a drug target against camel trypanosoma evansi. Pakistan Veterinary Journal 41: 235-241. https://doi.org/10.29261/pakvetj/ 2021.015

Kandeel, M., Dalab, A., Al-Shabebi, A. \& Al-Taher, A. (2020). Bioinformatics of pyrimidine metabolism in camels and Trypanosoma evansi: Uridine 5'-Diphosphate (UDP) Metabolic pathways and targeting atp diphosphatase. Journal of Camel Practice and Research 27: 145-158. https:// doi.org/10.5958/2277-8934.2020.00022.3

Kandeel, M. \& Kitade, Y. (2011a). Binding dynamics and energetic insight into the molecular forces driving nucleotide binding by guanylate kinase. Journal of molecular recognition : JMR 24: 322-332. https://doi.org/ 10.1002/jmr.1074

Kandeel, M. \& Kitade, Y. (2011b). The substrate binding preferences of Plasmodium thymidylate kinase. Biological and Pharmaceutical Bulletin 34: 173-176. https://doi.org/ 10.1248/bpb.34.173

Kanehisa, M., Araki, M., Goto, S., Hattori, M., Hirakawa, M., Itoh, M., Katayama, T., Kawashima, S., Okuda, S. \& Tokimatsu, T. (2007). KEGG for linking genomes to life and the environment. Nucleic Acids Research 36: 480-484. https://doi.org/10.1093/nar/gkm882

Kanehisa, M., Furumichi, M., Tanabe, M., Sato, Y. \& Morishima, K. (2016). KEGG: new perspectives on genomes, pathways, diseases and drugs. Nucleic Acids Research 45: D353-D361. https://doi.org/10.1093/nar/gkw1092
Kanehisa, M. \& Goto, S. (2000). KEGG: kyoto encyclopedia of genes and genomes. Nucleic Acids Research 28: 27-30. https://doi.org/10.1093/nar/28.1.27

Kato, A., Yasuda, Y., Kitamura, Y., Kandeel, M. \& Kitade, Y. (2012). Carbocyclic thymidine derivatives efficiently inhibit Plasmodium falciparum thymidylate kinase (PfTMK). Parasitology International 61: 501-503. https:// doi.org/10.1016/j.parint.2012.03.001

Labarga, A., Valentin, F., Anderson, M. \& Lopez, R. (2007). Web services at the European bioinformatics institute. Nucleic Acids Research 35: W6-W11. https://doi.org/10.1093/nar/ gkp302

Lee, C.S., Jimenez, B.M. \& O'Sullivan, W.J. (1988). Purification and characterization of uridine (thymidine) phosphorylase from Giardia lamblia. Molecular and Biochemical Parasitology 30: 271-277. https://doi.org/10.1016/0166-6851(88)90096-5

Madden, T. (2013). The BLAST sequence analysis tool, In: The NCBI Handbook [Internet]. 2nd edition. US: National Center for Biotechnology Information.

Marchler-Bauer, A., Anderson, J.B., Cherukuri, P.F., DeWeeseScott, C., Geer, L.Y., Gwadz, M., He, S., Hurwitz, D.I., Jackson, J.D. \& Ke, Z. (2005). CDD: A Conserved Domain Database for protein classification. Nucleic Acids Research 33: D192D196. https://doi.org/10.1093/nar/gki069

Noguchi, Y., Yasuda, Y., Tashiro, M., Kataoka, T., Kitamura, Y., Kandeel, M. \& Kitade, Y. (2013). Synthesis of carbocyclic pyrimidine nucleosides and their inhibitory activities against Plasmodium falciparum thymidylate kinase. Parasitology International 62: 368-371. https://doi.org/ 10.1016/j.parint.2013.03.009

Ogata, H., Goto, S., Fujibuchi, W. \& Kanehisa, M. (1998). Computation with the KEGG pathway database. Biosystems 47: 119-128. https://doi.org/10.1016/S0303-2647(98)000173

Sievers, F. \& Higgins, D.G. (2014). Clustal Omega, accurate alignment of very large numbers of sequences. In: Multiple sequence alignment methods. Springer, pp. 105116.

Silva, R.G., Kipp, D.R. \& Schramm, V.L. (2012). Constrained bonding environment in the Michaelis complex of Trypanosoma cruzi uridine phosphorylase. Biochemistry 51: 6715-6717. https://doi.org/10.1021/bi300914q

Silva, R.G., Vetticatt, M.J., Merino, E.F., Cassera, M.B. \& Schramm, V.L. (2011). Transition-state analysis of Trypanosoma cruzi uridine phosphorylase-catalyzed arsenolysis of uridine. Journal of the American Chemical Society 133: 9923-9931. https://doi.org/10.1021/ja2031294

Vanhollebeke, B., Truc, P., Poelvoorde, P., Pays, A., Joshi, P.P., Katti, R., Jannin, J.G. \& Pays, E. (2006). Human Trypanosoma evansi infection linked to a lack of apolipoprotein L-I. The New England Journal of Medicine 355: 2752-2756. https:/ /doi.org/10.1056/nejmoa063265

Wang, L., Schmidl, S.R. \& Stulke, J. (2014). Mycoplasma pneumoniae thymidine phosphorylase. Nucleosides Nucleotides Nucleic Acids 33: 296-304. https://doi.org/ 10.1080/15257770.2013.853783

Waterhouse, A., Bertoni, M., Bienert, S., Studer, G., Tauriello, G., Gumienny, R., Heer, F.T., de Beer, T.A.P., Rempfer, C. \& Bordoli, L. (2018). SWISS-MODEL: homology modelling of protein structures and complexes. Nucleic Acids Research 46: W296-W303. https://doi.org/10.1093/nar/gky427 